\title{
Flexible Facility Interior Layout: A Real Options Approach
}

\author{
Tong Zhao*and Chung-Li Tseng ${ }^{\dagger}$
}

\begin{abstract}
This paper explores a case of flexible facility layout, in which the layout process can be carried out in a dynamic manner responding to changing demands. We consider a facility such as an office building that needs to partition its interior space into units of different sizes and allocate them to different users. Facing different demands, each of which requires a different space size, the management of the facility needs to constantly exercise managerial real options, such as partitioning a big unit to small units and merging small units to a big one. Modeling the demand uncertainties as stochastic processes, we use the Monte Carlo simulation and solve rolling two-stage stochastic programs over time to value the flexible interior layout. Through numerical tests, we demonstrate that effectively and timely exercising the managerial options can significantly increase profit and service quality. Focusing on the dynamic behavior of the facility interior layout, the proposed options-based approach aims to improve facility management by proactive decision strategies. The approach may also be extended to facility expansion, maintenance and rehabilitation to increase service life and maintain service quality.

Keywords: Facility, interior layout, real options, stochastic model, Monte Carlo simulation, integer programming

\footnotetext{
${ }^{*}$ Corresponding author: Consultant, Delta Consulting Group, Inc., 310 Commerce Street, Occoquan, VA 22125, USA. Tel: (703) 497-7735, fax: (703) 497-7736, email: tzhao@delta-cgi.com

${ }^{\dagger}$ Associate Professor, Department of Engineering Management, University of Missouri-Rolla, Rolla, MO 65409, USA. Tel: (573) 341-7621, fax: (573) 341-6567, email: chungli@umr.edu
} 


\section{INTRODUCTION}

In a fast-changing world, a facility may find itself in a situation that its existing functions have become out of date and/or requires changes, which may impair its services. Facility layout, in terms of the arrangement of space and entities, reflects the designed functions of a facility. Intuitively, it may be desirable that the facility layout can be flexible in the sense that the layout can be adapted to changing circumstances. However, most layout changes may involve complicated design modifications, which may result in significant costs or require a long processing time, such that a flexible setting may not be feasible. This paper explores a case of facility interior layout, in which the layout process indeed can be carried out in a dynamic manner responding to changing demands.

The interior layout concerned in this paper refers to partitioning interior space into units of different sizes and allocating them to different entities (users). An office building is a good example. Companies of various sizes may want to lease an office space of a different size. In reality, space partitioning within an office building is usually handled by laying non-load-bearing walls or other partitions, which can be erected, moved or removed with little effort compared with the works involved in constructing the facility. In this situation, the interior layout can be changed easily within a short time. In this paper, we take a real-options approach to value such flexible facility interior layout. The layout process involves recurring decision making during the operational phase of the facility. With the evolution of underlying uncertainties, such as user demands, modeled by stochastic processes, three different space assignment strategies will be evaluated.

The concept of real options, stemming from financial options, refers to flexibility embedded in real operational processes, activities, or investment opportunities that are not financial instruments, in the sense that the decision maker (DM) has the right, but not the obligation to take a certain action (e.g., Trigeorgis 1996). Similar to the financial options case, where the DM exercises the options contingent upon stock price movements, we consider a case that the DM makes timely changes of the interior layout as the user demands evolve. That is, we treat the layout decision as an American-style real option (or flexibility). In this paper, these two terms, real option(s) and flexibility, are viewed equivalent and will be used interchangeably. 
Traditional facility layout problems, referring to activities that optimally arrange facilities or entities within a facility with regard to chosen criteria, are well researched (Heragu 1997). They are somewhat different from the interior layout problem considered in this paper. In the traditional facility layout problems, the criteria are usually to minimize the total weighted distance between entities, in which weights represent adjacency priorities or cost related to material flow volume (Francis et al. 1992). Researchers have developed different models for the facility layout problem, for example, the quadratic assignment problem (QAP) (Koopmans and Beckmann 1957), the linear mixed-integer programming model (Love and Wong 1976), and the quadratic set covering model (Bazaraa and Goode 1975). These models, such as QAP, are NP-complete, which receive most research attentions in developing and improving heuristics. Recently, researchers start to address dynamic nature of facility layout problem. For example, Lacksonen (1994) provides a general dynamic layout algorithm combining the QAP and mixed-integer programming. However, most researchers make the assumption that the change of requirement is known at the time the first design decision is made, which may not be realistic (Heragu and Kochhar 1994).

In this paper, we take a different treatment on the layout problem. Instead of focusing on the layout problem in the design phase as most traditional approaches do, we focus on the operational phase. Therefore, we consider the problem over the entire operational period of the facility. From the real options perspective, the managerial decisions considered in the paper are real options, including rejecting a user, partition a big unit to small ones, merging small units to a big one, and relocating existing users. We do not formulate this problem by a multi-stage stochastic program because it is computationally intractable. Instead we use the Monte Carlo (MC) simulation and solve rolling two-stage problems over time. Our main interest is to value these managerial real options, i.e., to see how much each managerial option contribute to the overall profits, which helps devise good operational strategies.

Through numerical tests, we demonstrate that effectively and timely exercising the managerial options can significantly increase profit and service quality. Focusing on the dynamic behavior of the facility interior layout, the proposed options-based approach aims to improve facility management by proactive decision strategies. The approach may also 
be extended to facility expansion, maintenance and rehabilitation to increase service life and maintain service quality.

The major contribution of this paper is to demonstrate the value of managerial flexibility in facility layout and to propose an approximation method in solving a complex multi-stage stochastic problem with a large number of integer decision variables involved. From the computational perspective, real options valuation is integrated with integer programming, which has not yet be attempted before in literature.

\section{VALUING FLEXIBILITY IN INTERIOR LAYOUT}

In this section, a facility is viewed as a dynamic system, in which managerial flexibility is available for the DM to make contingent decisions. In order to maximize the (expected) profit, uncertainties, such as demands of different users and user's occupancy duration, must be considered. With the managerial flexibility, the uncertainties may be turned to profitable opportunities. To demonstrate the value of flexible interior layout, we consider a facility that is an office building. Assume that the facility can accommodate two types of demand of office space, big units (type A) and small units (type B).

Suppose that there are two main types of facility users, type A and type B. Type A users rent (type A) big units, and each big unit consists of (or is equivalent to) $m_{B}$ conjoint (type B) small units. Type B users rent the small units. For simplicity, the big unit and the small unit will be called type A and type B unit, respectively. Assume all users arrive only at the beginning of a time period. All users must sign and fulfill a rental lease that requires a minimum duration of residence. To add some variation, with some probability each user may terminate the lease earlier by paying some fine. For simplicity, it is assumed that the facility only takes demands once (in the beginning of) a year. If a user would like to stay more than the term, she is considered as a new demand as the lease expires. For simplicity, it is also assumed that the facility has been designed to have $m_{A}$ identical type A units and each type A unit consists of $m_{B}$ (standard) type B units. Figure 1 illustrates an example of such a facility with $m_{A}=3$ and $m_{B}=4$. 


\section{Managerial flexibility}

The managerial flexibility considered here includes the following options:

1. (Partition) The DM may partition a type A unit to several small and adjacent type B units as defined above.

2. (Merge) The DM may merge $m_{B}$ small and adjacent type B units to a type A unit (an exactly reverse process of the partition option).

3. (Reject) The DM may reject a user for strategic reasons. For example, the DM may reject type B users in anticipation of accommodating type A users, or vice versa.

4. (Relocate) In order to merge small units, the DM may relocate a continuing (type B) user to another equivalent unit with a compensation to the user.

5. (Promote with a discount) The DM can promote the units that would otherwise be vacant with a discounted rate. The promotion is normally subject to more restricted terms than in the regular lease.

To model the promotion option, in addition to the regular users (types A and B), we assume there is another type of users, called type C, who are willing to sign a lease for a type $\mathrm{B}$ unit with more restricted terms under a discounted rental rate. This may be viewed as another form of demand elasticity.

\section{Problem formulation}

The facility layout decisions are made at discrete time points. The timing of event occurrence is as follows: at time $t$, some users leave and new users arrive. Upon receiving the information on the demands and current occupancy status of the facility, the DM maximizes the (expected) net profit by taking or rejecting new users, relocating continuing users and assigning units to new users. The step for assigning units may implicitly involve partitioning and/or merging units, which are assumed to be handled instantaneously with a negligible cost. This process repeats at time $t+1$, and continues till time $T$.

Three optimization models that support the DM's unit assignment decision-making are considered. Model 1 is the most straightforward one. Upon receiving all requests, the DM assigns units based on available vacancy without using the relocation option. In 
Model 2, the DM may exercise the relocation option to better assign units and achieve a higher profit. Both Models 1 and 2 are deterministic. Model 3 is a two-stage stochastic one. The DM looks ahead one more time period and performs the optimization with all available options. The following standard notation will be used herein.

$m_{A}$ : total number of type A units in the facility.

$i$ : index of type A units in the facility, $i=1, \cdots, m_{A}$.

$m_{B}$ : total number of type B units within each type A unit.

$j$ : index of type B unit within each type A unit, $j=1, \cdots, m_{B}$.

$t$ : time index, $t=1, \cdots, T$, where $T$ is the length of the time horizon considered.

$x_{i}^{t}: 0 / 1$ decision variable indicating whether the $i$ th type A unit will be assigned to a type A user in time period $t$.

$x_{i}^{t, \text { old }}: 0 / 1$ decision variable indicating whether the $i$ th type A unit will be assigned to an existing type A user in time period $t$.

$x_{i}^{t, \text { new }}: 0 / 1$ decision variable indicating whether the $i$ th type A unit will be assigned to an incoming type A user in time period $t$.

$y_{i j}^{t}: 0 / 1$ decision variable indicating whether the $j$ th type $\mathrm{B}$ unit within the $i$ th type A will be assigned to a type B user in time period $t$.

$y_{i j}^{t \text {,old }}: 0 / 1$ decision variable indicating whether the $j$ th type $\mathrm{B}$ unit within the $i$ th type A will be assigned to an existing type B user in time period $t$.

$y_{i j}^{t \text {,new }}: 0 / 1$ decision variable indicating whether the $j$ th type B unit within the $i$ th type A will be assigned to an incoming type B user in time period $t$.

$z_{i j}^{t}: 0 / 1$ decision variable indicating whether the $j$ th type B unit within the $i$ th type A unit will be assigned to a type $\mathrm{C}$ user in time period $t$.

$z_{i j}^{t, \text { old }}: 0 / 1$ decision variable indicating whether the $j$ th type B unit within the $i$ th type A unit will be assigned to an existing type $\mathrm{C}$ user in time period $t$.

$z_{i j}^{t, \text { new }}: 0 / 1$ decision variable indicating whether the $j$ th type $\mathrm{B}$ unit within the $i$ th type A unit will be assigned to an incoming type $\mathrm{C}$ user in time period $t$. 
$u_{i j}^{t}$ : state variable indicating how long the $j$ th type B unit within the $i$ th type A unit has been occupied by the end of time period $t$.

$v_{i}^{t}$ : state variable indicating how long the $i$ th type A unit has been occupied (by a type A user) by the end of time period $t$.

$D_{A}^{t}$ : new demand of type A units in period $t$.

$D_{B}^{t}$ : new demand of type B units from type B users in period $t$.

$D_{C}^{t}$ : new demand of type B units from type C users in period $t$.

$R_{A}^{t}$ : constant rent rate for a type A unit in period $t$, which may change over time.

$R_{B}^{t}$ : constant rent rate for a type $\mathrm{B}$ unit (for a type B user) in period $t\left(R_{A}^{t}>m_{B} R_{B}^{t}\right)$.

$R_{C}^{t}$ : constant rent rate for a type $\mathrm{B}$ unit (for a type $\mathrm{C}$ user) in period $t\left(R_{B}^{t}>R_{C}^{t}\right)$.

$c_{B}^{t}$ : constant compensation for a type $\mathrm{B}$ user in period $t$, if (i) the user is a continuing user; and (ii) the user is relocated by the management to another unit in time period $t$. The amount of compensation may change over time. (Note that the compensation for relocating a user may also be modeled as a function of distance relocated, which can be easily included in the proposed model, at, however, the price of increased complexity.)

$I_{A}^{t}$ : index set identifying all vacant type A units at time $t$.

$I_{B}^{t}$ : index set identifying all vacant type B units at time $t$.

$J^{t}(i)$ : index set identifying vacant type B units in the $i$-th type A unit.

$n:$ model index, and $n=1,2,3$.

$\left(P_{n}^{t}\right)$ : three proposed model formulations, $n=1,2,3$.

$\mathbf{X}^{t}$ : collection of all decision variables at time $t$.

$\xi_{t}$ : collection of demand uncertainties at time $t$.

$f_{t}(\cdot)$ : net revenue function in period $t$.

$l$ : index variable for user type, $l \in\{\mathrm{A}, \mathrm{B}, \mathrm{C}\}$.

$\epsilon_{l}:$ a standard normal random variable for type $l$ user.

$\mu_{l}$ : drift of the demand process for type $l$ user. 
$\sigma_{l}$ : volatility of the demand process for type $l$ user.

$p_{l}$ : probability associated with a binomial scenario branch for demand $l$.

$r:$ discount rate.

$S$ : number of possible scenarios for each time period.

$k$ : ordinal number for a simulation run.

$K$ : total number of the simulation runs.

$\mathrm{NPV}_{n}^{(k)}$ : NPV of the $k$-th simulation run using Model $\left(P_{n}^{t}\right)$.

$F_{n}^{*}$ : the expected value of the total profit using Model $\left(P_{n}^{t}\right)$.

Define the index sets for available units as follows:

$$
\begin{gathered}
I_{A}^{t} \equiv\left\{i \mid v_{i}^{t-1}=0\right\}, \\
I_{B}^{t} \equiv\left\{(i, j) \mid u_{i j}^{t-1}=0\right\},
\end{gathered}
$$

and

$$
J^{t}(i) \equiv\left\{j \mid u_{i j}^{t-1}=0 \text { and } v_{i}^{t-1}=0\right\} .
$$

The index sets $I_{A}^{t}$ and $I_{B}^{t}$ identify all vacant type A and type B units at time $t$, respectively. The set $J^{t}(i)$ only identifies vacant type B units in the $i$-th type A unit.

\section{Model 1: Deterministic model without the relocation option}

In this model, the DM is assumed to be myopic. That means the DM does not look ahead and only maximizes the current time period. Furthermore, it is assumed that the DM does not have the relocation option. At the beginning of period $t$, based on available vacancy $\left(I_{A}^{t}, I_{B}^{t}\right.$, and $\left.J^{t}\right)$ the DM assigns new users to vacant units without relocating continuing users. The management will solve the following integer program, denoted by $\left(P_{1}^{t}\right)$, to determine unit assignments.

$\left(P_{1}^{t}\right)$

$$
\max \sum_{i=1}^{m_{A}} R_{A}^{t} x_{i}^{t}+\sum_{i=1}^{m_{A}} \sum_{j=1}^{m_{B}}\left(R_{B}^{t} y_{i j}^{t}+R_{C}^{t} z_{i j}^{t}\right)
$$


subject to

$$
\begin{gathered}
\sum_{i \in I_{A}^{t}} x_{i}^{t} \leq D_{A}^{t}, \\
\sum_{(i, j) \in I_{B}^{t}} y_{i j}^{t} \leq D_{B}^{t}, \\
\sum_{(i, j) \in I_{B}^{t}} z_{i j}^{t} \leq D_{C}^{t}, \\
x_{i}^{t}+\sum_{j \in J^{t}(i)}\left(y_{i j}^{t}+z_{i j}^{t}\right) \leq 1, \forall i, \\
x_{i}^{t}=1, \text { if } i \notin I_{A}^{t}, \\
y_{i j}^{t}=1, \text { if }(i, j) \notin I_{B}^{t}, \\
x_{i}^{t}, y_{i j}^{t}, z_{i j}^{t}, \quad \in\{0,1\} \forall i, \forall j .
\end{gathered}
$$

Equations (3) - (5) ensure that the demands are served by available units. Constraint (6) imposes that a unit cannot be assigned to more than one user simultaneously. Equations (7) and (8) are used in the objective function. After solving $\left(P_{1}^{t}\right)$, the state variables $v_{i}^{t}$ and $u_{i j}^{t}$ are updated by

$$
v_{i}^{t}=\left(v_{i}^{t-1}+1\right) x_{i}^{t}
$$

and

$$
u_{i j}^{t}=\left(u_{i j}^{t-1}+1\right) y_{i j}^{t}
$$

Accordingly, the index sets for the availability of the units $I_{A}^{t}, I_{B}^{t}$ and $J^{t}$ can be updated for the next time period.

\section{Model 2: Deterministic model with the relocation option}

In this model, the DM is still myopic but has the relocation option. At the beginning of period $t$, the DM assigns new users to vacant units and the continuing users (type B) may be relocated if doing so can make a type A unit available for a type A user. The DM may profit by exercising the relocation option because $R_{A}^{t}>m_{B} R_{B}^{t}$. In the formulation, because of the relocation each decision variable will be split to two. For example, $x_{i}^{t}$ is split

to $x_{i}^{t, \text { new }}$ and $x_{i}^{t, \text { old }}$. The variables marked 'new' are used to satisfy the 'new' (incoming) demand; those marked 'old' are used to seek a profitable relocation while satisfying the 
'old' demand (existing tenants). Before solving the problem, it is assumed that the unit occupancy statuses $\left(x_{i}^{t-1}, y_{i j}^{t-1}\right.$, and $\left.z_{i j}^{t-1}\right)$ from the previous time period are known (also defined later for their update). The integer program formulation, denoted as $\left(P_{2}^{t}\right)$, is presented below.

$\left(P_{2}^{t}\right)$

$$
\begin{gathered}
\max \sum_{i=1}^{m_{A}} R_{A}^{t}\left(x_{i}^{t, \text { new }}+x_{i}^{t, \text { old }}\right)+\sum_{i=1}^{m_{A}} \sum_{j=1}^{m_{B}} R_{B}^{t}\left(y_{i j}^{t, \text { new }}+y_{i j}^{t, \text { old }}\right)+ \\
R_{C}^{t}\left(z_{i j}^{t, \text { new }}+z_{i j}^{t, \text { old }}\right)-\frac{c_{B}}{2} \sum_{i=1}^{m_{A}} \sum_{j=1}^{m_{B}}\left(\left|y_{i j}^{t, \text { old }}-y_{i j}^{t-1}\right|+\left|z_{i j}^{t, \text { old }}-z_{i j}^{t-1}\right|\right)
\end{gathered}
$$

subject to

$$
\begin{gathered}
\sum_{i=1}^{m_{A}} x_{i}^{t, \text { old }}=\sum_{i=1}^{m_{A}} x_{i}^{t-1}, \\
\sum_{i=1}^{m_{A}} \sum_{j=1}^{m_{B}}\left(y_{i j}^{t, \text { old }}+z_{i j}^{t \text {,old }}\right)=\sum_{i=1}^{m_{A}} \sum_{j=1}^{m_{B}}\left(y_{i j}^{t-1}+z_{i j}^{t-1}\right), \\
\sum_{i=1}^{m_{A}} x_{i}^{t, \text { new }} \leq D_{A}^{t}, \\
\sum_{i=1}^{m_{A}} \sum_{j=1}^{m_{B}} y_{i j}^{t, \text { new }} \leq D_{B}^{t}, \\
\sum_{i=1}^{m_{A}} \sum_{j=1}^{m_{B}} z_{i j}^{t, \text { new }} \leq D_{C}^{t}, \\
x_{i}^{t, \text { new }}+\sum_{i=1}^{m_{A}} \sum_{j=1}^{m_{B}}\left(y_{i j}^{t, \text { new }}+y_{i j}^{t, \text { old }}+z_{i j}^{t, \text { new }}+z_{i j}^{t, \text { old }}\right) \leq 1, \forall i, \\
x_{i}^{t \text {,old }}+x_{i}^{t, \text { new }} \leq 1, \\
y_{i j}^{t, \text { old }}+y_{i j}^{t, \text { new }} \leq 1, \\
z_{i j}^{t, \text { old }}+z_{i j}^{t, \text { new }} \leq 1, \\
x_{i}^{t, \text { old }}, x_{i}^{t, \text { new }}, y_{i j}^{t \text {,old }}, y_{i j}^{t, \text { new }}, z_{i j}^{t, \text { old }}, z_{i j}^{t, \text { new }}, \quad \in\{0,1\} \forall i, \forall j .
\end{gathered}
$$

It can be seen that (13) and (14) ensure that existing tenants are covered regardless of the relocation process. Constraints $(15)$ - (18) correspond to $(3)$ - (6) in $\left(P_{1}^{t}\right)$. When a relocation is made, $y_{i j}^{t-1} \neq y_{i t}^{t \text {,old }}$ or $z_{i j}^{t-1} \neq z_{i t}^{t \text {,old }}$ and a relocation cost is applied in the objective function. After solving $\left(P_{2}^{t}\right)$, the following variables are updated for the iteration of the next time period.

$$
x_{i}^{t}=x_{i}^{t, \text { old }}+x_{i}^{t, \text { new }}
$$




$$
\begin{aligned}
& y_{i j}^{t}=y_{i j}^{t, \text { old }}+y_{i j}^{t, \text { new }} \\
& z_{i j}^{t}=z_{i j}^{t, \text { old }}+z_{i j}^{t, \text { new }}
\end{aligned}
$$

If unit relocation does occur, the state variables $u_{i j}^{t}$ and $v_{i}^{t}$ corresponding to units relocated should also be modified and updated accordingly.

Note that the last two terms (involving absolute values) in the objective function (12), though nonlinear, can be converted to a linear model. Therefore, $\left(P_{2}^{t}\right)$ remains an integer linear program.

\section{Model 3: Two-stage stochastic model}

In this stochastic model, at each time period the DM looks ahead one more period and performs the optimization. The DM will forecast the demand for the next period. Based on the forecast and the current information of the demand, the DM makes decisions to maximize the expected profit. At the beginning of period $t$, the DM assigns new users to vacant units. If necessary, continuing users may be relocated. Let $\mathbf{X}^{t}$ be the collection of all decision variables in $\left(P_{2}^{t}\right), x_{i}^{t \text {,old }}, x_{i}^{t, \text { new }}, y_{i j}^{t \text {,old }}, y_{i j}^{t, \text { new }}, z_{i j}^{t \text {,old }}$, and $z_{i j}^{t \text {,new }}$. Also let $\xi_{t}$ be the collection of demand uncertainties at time $t, D_{A}^{t}, D_{B}^{t}$, and $D_{C}^{t}$. Denote the objective function of $\left(P_{2}^{t}\right)$ as $f_{t}\left(\mathbf{X}^{t} ; x_{i}^{t-1}, y_{i j}^{t-1}, z_{i j}^{t-1}, \xi_{t}\right)$, a function of $\mathbf{X}^{t}$ given the initial state values

of $x_{i}^{t-1}, y_{i j}^{t-1}$ and $z_{i j}^{t-1}$, and the demand realization $\xi_{t}$ at time $t$. Also denote the collection of the constraints of $\left(P_{2}^{t}\right),(13)$ - $(25)$, as $\left(Q_{t}\right)$, parametric in $t$. The two-stage formulation, denoted by $\left(P_{3}^{t}\right)$, is presented as follows:

$\left(P_{3}^{t}\right)$

$$
\max f_{t}\left(\mathbf{X}^{t} ; x_{i}^{t-1}, y_{i j}^{t-1}, z_{i j}^{t-1}, \xi_{t}\right)+e^{-r} E_{t}\left[f_{t+1}\left(\mathbf{X}^{t+1} ; x_{i}^{t}, y_{i j}^{t}, z_{i j}^{t}, \xi_{t+1}\right)\right]
$$

subject to constraints $\left(Q_{t}\right)$ and $\left(Q_{t+1}\right)$. In (26), $r$ is the risk-adjusted discount rate for each time period, which accounts both for the time value of money and for the DM's risk preference. The expectation operator in (26) is not measured in the risk-neutral framework commonly adopted in financial option valuation. This is due to the fact that there are no traded derivative securities dependent on the values of the underlying demand uncertainties.

To solve $\left(P_{3}^{t}\right)$, if the demand uncertainty can be modeled by scenarios, the stochastic formulation can be converted to a deterministic equivalent integer program. (The de- 
mand uncertainty modeling will be presented in the next section.) This conversion will increase the number of variables and constraints of the original formulation. In this case, these increased variables and constraints are associated with different scenarios in the second-stage problem. The conversion can be generally applied to a multi-stage model, in which the DM looks ahead multiple periods. However, the number of scenarios normally increases exponentially as the number of stages increases. In this research, in addition to the demand uncertainty we also consider the fact that a tenant may early-terminate his lease. Due to the computational feasibility, the two-stage model seems the only stochastic model that can be handled realistically.

To summarize, all three models presented are either linear integer programs (ILPs) or can be converted to equivalent ILPs. They can all be solved by commercial ILP solver such as CPLEX.

\section{Uncertainty modeling}

The arrival of each demand type (A, B, or C) is modeled as a stochastic process. First define a stochastic process $\left\{d_{l}^{t}\right\}_{t=1}^{T}$, whose evolution follows the following relation:

$$
\frac{d_{l}^{t+\Delta t}-d_{l}^{t}}{d_{l}^{t}}=\mu_{l} \Delta t+\sigma_{l} \epsilon_{l} \sqrt{\Delta t}, l \in\{\mathrm{A}, \mathrm{B}, \mathrm{C}\}
$$

where $\mu_{l}$ and $\sigma_{l}$ represent the drift and the volatility for demand $l$, respectively, and $\epsilon_{l}$, $l \in\{\mathrm{A}, \mathrm{B}, \mathrm{C}\}$, is a standard normal random variable, independent of time and $l$. The time step is denoted by $\Delta t$. Since the demand process generated by (27) is not integral, its values are rounded off to a nearest integer before it can be used.

$$
D_{l}^{t} \leftarrow\left\lfloor d_{l}^{t}+0.5\right\rfloor, t \in\{0,1,2, \cdots, T\}, l \in\{\mathrm{A}, \mathrm{B}, \mathrm{C}\},
$$

The demand model $d_{l}^{t}, l \in\{\mathrm{A}, \mathrm{B}, \mathrm{C}\}$, in (27) is the discrete-time model of the so-called geometric Brownian motion (GBM), which is commonly used in finance to model stock price movements. It's evolution can be approximated by a binomial branch (e.g., Hull 1999, Luenberger 1998): given current demand $d_{l}^{t}$, in the next time period $t+\Delta t$ two states are possible: the up state $d_{l, \text { up }}^{t+1}=$ up_factor $\cdot d_{l}^{t}$ with probability $p_{l}$ and the down state $d_{l, \mathrm{dn}}^{t+1}=$ down_factor $\cdot d_{l}^{t}$ with probability $1-p_{l}$, where

$$
\text { up_factor }=\exp \left(\sigma_{l} \sqrt{\Delta t}\right)=\frac{1}{\text { down_factor }}
$$


and

$$
p_{l}=\frac{\exp \left(\mu_{l} \Delta t\right)-\text { down_factor }}{\text { up_factor }- \text { down_factor }}, l \in\{\mathrm{A}, \mathrm{B}, \mathrm{C}\} .
$$

An example of a binomial branching is given in Fig. 2. Equation (28) is applied to each node of the branch to round off $d_{l}^{t}$ to $D_{l}^{t}$.

Given a realization of the three demand uncertainties $d_{l}^{t}, l=\mathrm{A}, \mathrm{B}, \mathrm{C}$ at time $t$, there are three independent binomial branches (assuming $\epsilon_{l}, l=\mathrm{A}, \mathrm{B}, \mathrm{C}$, are mutually

independent). Equivalently, there are $2^{3}=8$ possible scenarios for $\left(D_{A}^{t+1}, D_{B}^{t+1}, D_{C}^{t+1}\right)$ in the next time period $t+1$. If early termination of the lease is considered, the number of possible scenarios, denoted by $S$, for the next time period is bounded from above by $2^{m_{A} m_{B}+3}$, i.e.,

$$
S \leq 2^{m_{A} m_{B}+3}
$$

In this situation, each scenario not only describes a set of demand $\left(D_{A}^{t+1}, D_{B}^{t+1}, D_{C}^{t+1}\right)$ in the next time period $t+1$, but also additional spaces that become available. Based on the scenarios, the two-stage stochastic program $\left(P_{3}^{t}\right)$ in Model 3 can be converted to an equivalent deterministic ILP and be solved by CPLEX.

\section{Simulation algorithm}

For Models 1 to 3, the simulation algorithm is proposed below, where $k$ is the index for simulation iteration and $K$ is a predetermined maximal number of iterations.

\section{Algorithm}

Data: Model number $n \in\{1,2,3\}$ is given; $K$ : the total number of simulation iterations is given; $T$ : duration of facility life-cycle is given.

Step 0: $k \leftarrow 1$

Step 1: If $k>K$, go to Step 7. Otherwise $\operatorname{NPV}_{n}^{(k)} \leftarrow 0$.

Step 2: Set $t \leftarrow 0$, and set all variables (i.e., $x_{i}^{t}, y_{i j}^{t}, z_{i j}^{t}, u_{i j}^{t}, v_{i}^{t} \leftarrow 0$ ) to be zero.

Step 3: Generate the demand realization $\left(D_{A}^{t}, D_{B}^{t}\right.$, and $\left.D_{C}^{t}\right)$ based on the uncertainty model (27) - (28). (If $n=3$, also generate scenarios for $D_{A}^{t+1}, D_{B}^{t+1}$, and $D_{C}^{t+1}$ for the two-stage optimization.) Solve $\left(P_{n}^{t}\right)$ using CPLEX. Realize the optimal unit assignment decisions. Denote the optimal objective value by $h_{t}$. 
Step 4: Update the state variables $\left(u_{i j}^{t}\right.$ and $\left.v_{i}^{t}\right)$, and index sets $\left(I_{A}^{t}, I_{B}^{t}\right.$, and $\left.J^{t}\right)$.

Step 5: If $t \geq T$, go to Step 6. Otherwise, $t \leftarrow t+1$, and go to Step 3 .

Step 6: $\operatorname{NPV}_{n}^{(k)} \leftarrow \mathrm{NPV}_{n}^{(k)}+e^{-r} h_{t} ; k \leftarrow k+1$, go to Step 1 .

Step 7: $F_{n}^{*} \leftarrow \frac{1}{K} \sum_{k=1}^{K} \mathrm{NPV}_{n}^{(k)}$

In Step $7, F_{n}^{*}(n=1,2,3)$ is the expected NPV of the total profit of the facility operation using Model $n$ for dynamic layout.

Overall, the algorithm simulates the facility operations forward in time during the operational phase. At each time period, the DM makes the layout decisions based on one of the three models proposed. The decisions made at time $t$ becomes the initial condition of the model at $t+1$. Then the simulation proceeds till the end of planning horizon.

As mentioned previously, Models 1 to 3 represent different strategies for decisionmaking at each time point. By comparing Models 1 and 2,

$$
F_{2}^{*}-F_{1}^{*}=\text { the value of the relocation option; }
$$

comparing Models 2 and 3,

$$
F_{3}^{*}-F_{2}^{*}=\text { the value of performing stochastic optimization; }
$$

and comparing Models 1 and 3,

$$
F_{3}^{*}-F_{1}^{*}=(\text { a lower bound of }) \text { the value of dynamic facility layout. }
$$

Note that because Model 3 employs two-stage, instead of multistage, optimization, the option value obtained in (34) is only a lower bound.

\section{Model complexity}

A comparison of the complexity, in terms of the numbers of variables and constraints, of the models is summarized in Table 1. Since the objective function of Model $\left(P_{2}^{t}\right)$ is not exactly linear, which, however, can be converted to linear, its complexity in Table 1 includes the additional variables and constraints required for the conversion. It can be seen that Model $\left(P_{3}^{t}\right)$ has the highest complexity, followed by Models $\left(P_{2}^{t}\right)$, and then by $\left(P_{1}^{t}\right)$, in terms of the number of variables and the number of constraints. If the number of 
scenarios for each time period in Model $\left(P_{3}^{t}\right)$ is fixed (say, $S=2^{3}$ without considering early termination of the lease), the complexity is a polynomial of $m_{A}$ and $m_{B}$, the numbers of the units. But if early termination of the lease is considered, the number of (worst-case) scenarios $S$ increases exponentially (31), so do the numbers of variables and constraints. This is reasonable because an early termination of the lease creates an additonal avaiable space, which may affect the optimal interior layout decision.

If the DM is to look ahead two further periods and perform the optimization (i.e., a three-stage model), the complexity would be even higher. For example, assume $m_{A}=3$ and $m_{B}=4$, then $S=2^{15}$. The number of variables and the number of constraints in $\left(P_{3}^{t}\right)$ are around 23,000 times $\left(\approx\left(S^{2}+S+1\right) /(S+1)\right)$ as many as those in $\left(P_{2}^{t}\right)$. This becomes too intractable to solve within an acceptable time with existing computing resources. Detailed information regarding computational time will be presented in the next section of numerical tests. Therefore, in our numerical tests we focus on two-stage models for $\left(P_{3}^{t}\right)$.

\section{Comparison with existing models}

In this section we compare the proposed model with existing methods in literature (e.g., to name a few, Lacksonen 1994 and Kochhar and Heragu 1999) for solving the facility layout problems in terms of computational complexity. It should be noted that these existing methods, though have been commonly applied in dynamic environments, are deterministic approaches. Typically, in the first stage these methods estimate rearrangement costs and determine stationary departments, based on which the second stage problem does the layout optimization. Therefore, we shall compare our proposed Model 2, a deterministic model with the reallocation option, with the second stage problem of the existing methods. It is fair to say that in this paper we consider a special case of the general facility layout problems. Due to our problem setup, basic facility units have been fixed (including both size and locations) and our problem is to group adjacent small units to a bigger unit. So we formulate the problem as a binary integer linear program. Whereas, the general facility layout problems can freely determine the size of each rectangular de-

partment on the $R^{2}$ space. Therefore, the general layout formulation requires much more 
variables and constraints than our formulation. For example, for a single unit, we use one single binary variable to indicate its occupancy status. But the general layout formation will require at least six variables and four linear constraints to describe a rectangular department, whose area roughly matches a given value (see P. 62 of Lacksonen 1994). If the required precision of the area matching is high, more variables and constraints will be needed. Furthermore, in the general layout formulation the so-called non-overlapping constraints that prevent two departments in the same time overlap must be imposed to each pair of the departments, incurring either nonlinear constraints or as many as five linear constraints plus four binary variables, as in Montreuil (1990), for each pair of departments. The non-overlapping constraints are deemed the most challenging part of the layout formulation. This is avoided in our setup formulation. Therefore, it can be seen that the computational burden for our approach is much lighter than the general facility layout problems. That is why we can incorporate the deterministic layout optimization into the stochastic framework using Monte Carlo simulations and obtain results within reasonable CPU times.

\section{NUMERICAL TESTS}

In our numerical tests, a facility with $m_{A}$ big units is considered. Assume that the regular lease terms are 2 years for types $\mathrm{A}$ and $\mathrm{B}$ users, and 1 year for type $\mathrm{C}$ users. Also assume that some type B users may terminate the lease earlier after one-year stay with probability 0.2 . That is, such type B users would have probability 0.8 to fulfill the lease of two-year term. The exact number of such type B users is predetermined. However, the exact users who may terminate the lease early are generated randomly. Assume the maintenance cost for the space equivalent to a type B unit is $\$ 2,000$ per year. All other parameters are summarized in Table 2 .

\section{Computational time}

First we test the computational time. A numerical example with $m_{A}=3$ and $m_{B}=$ 4. Assume that in each time period there are two type B users who may terminate the lease early. So $S=2^{3+2}=32$. The average number of variables and the average 
number of constraints involved in 100 consecutive simulation runs with $T=25$ (years) are summarized in Table 3, along with the CPU times. Note the CPU times in Table 3 are the total times for the MC simulation including 100 runs, with each run simulating the operation of the facility over the 25 -year lifecycle. Therefore, each simulation run involves solving $T(=25)$ ILPs using CPLEX. Since early termination for type B users may not necessarily be applicable for each time period (e.g., all occupants are type A), the numbers of variables and constraints are not necessarily the same from time to time. Table 3 reports the average numbers of variables and constraints for $\left(P_{n}^{t}\right), n=1,2,3$, in all time periods. It can be seen that Models $\left(P_{1}^{t}\right)$ and $\left(P_{2}^{t}\right)$ require little computational times. For Model $\left(P_{3}^{t}\right)$, each MC simulation run takes about 36 seconds, and the overall simulation terminates within around 1 hour, using a Pentium $500 \mathrm{MHz}$ personal computer. This computational time may be further reduced with a faster computer and/or more refined implementation. It can be used for facility planning and be used for approving/arranging lease applications, which normally do not require real-time response.

In the following numerical tests, we focus on the value of flexibiity and service quality. Six test cases corresponding to six different initial conditions, such as number of units and initial demand, are considered. In combination with the possibility of employing the promotion with a discount, there are totally twelve different test cases. The simulation algorithm proposed in the previous section is applied to the test problems. The three models $\left(P_{n}^{t}\right), n=1,2,3$, are tested in the twelve test cases. The test results are summarized in Tables 3 and 5 .

\section{Value of flexibility}

It can be seen from Tables 4 and 5, the profits obtained by Model 3 are always greater than those by Model 2, which are greater than those of Model 1 in all six test cases. Directly comparing Models 1 and 2 shows the value of the relocation option. The profit obtained by Model 2 is on average $2.8 \%$ higher than that obtained by Model 1 when the promotion of taking type $\mathrm{C}$ users is applied; $3.0 \%$ without the promotion. The differences between Models 2 and 3 show the value of using stochastic programming. The profit

obtained by Model 3 is on average $4.3 \%$ higher than that obtained by Model 2 with the 
promotion; $0.9 \%$ without the promotion. In addition, directly comparing the results of the same items in Tables 4 and 5, we can obtain the value of the promotion, which results in $1.7 \%, 1.3 \%$ and $4.7 \%$ profit increases on average for Models 1, 2 and 3, respectively. The difference between Model 1 in Table 5 and Model 3 in Table 4 shows the overall value of all real options considered, which on average accounts for $8.8 \%$ of the (expected) profit. Note that these tests only consider the benefit (profit) of the real options that can be transacted. If one would account for the opportunity profits, such as the loss of revenue, the difference will be even more significant. Besides the monetary benefits, the real options also result in improvement of service and occupancy rates of the facility, discussed in the next section.

\section{Service and occupancy rates of the facility}

Define the service rate for each demand type and the occupancy rate for each unit type as follows:

Service rate for demand (type A, B or C) $=$

$$
\frac{\text { Total demand (type A, B or C) serviced over }[0, T]}{\text { Total demand (type A, B or C) over }[0, T]}
$$

and

Occupancy rate for unit (type $\mathrm{A}$ or $\mathrm{B})=$

$$
\frac{\text { Number of units (type A or B) occupied over }[0, T]}{\text { Number of units (type A or B) available over }[0, T]}
$$

From the complement of the service rate for each demand type (service rate subtracted by one), one can see the percentage of the demand type that has been turned down (unserviced), which measures some extent of loss of profitable opportunities. For example, a type A user may not get serviced if the facility does not have $m_{B}$ conjoint small units, which may well be resolved by having the relocation option. The occupancy rate measures the efficiency of space use. The occupancy and service rates for each different type are summarized in Tables $6-14$.

From Tables $6,7,11$, and 12, it can be seen that exercising the real options increases the service rate for demand type A but decreases the service rate for demand type B. This 
is due to the assumption that $R_{A}^{t}>m_{B} R_{B}^{t}$, i.e., renting a type $\mathrm{A}$ unit is more profitable than renting $m_{B}$ small units (type B). Certainly, the optimization associated with the exercise of the real options imposes a higher priority for servicing demand type A than type B.

From Tables 9, 10, 13, and 14, a similar result as the service rate is obtained that Model 3 has a higher occupancy rate for type A unit than Model 2, which has a higher rate than Model 1. A reversed order is obtained for the type B unit.

From Table 8, it can be seen that the service rate for type $\mathrm{C}$ demand is very low due to $R_{A}^{t}>m_{B} R_{B}^{t}>m_{B} R_{C}^{t}$. By thoroughly examining Tables $6-14$, it can be seen that the introduction of type $\mathrm{C}$ users improves the total occupancy rate of the facility, but it has little influence on occupancy rate for type A units in Models 1 and 2. However, the introduction of type $\mathrm{C}$ users significantly increases the service rate of type $\mathrm{A}$ demand and occupancy rate of type A unit, but reduces the service rate of type B demand and occupancy rate of type B unit in Model 3. This result may not seem obvious since type C users occupy type B units. However, because the term difference in the leases, allocating a vacant type $\mathrm{A}$ unit by a type $\mathrm{C}$ user (1-year lease) turns out to be more profitable than by a type B user (2-year lease). Because the former makes the unit available in a year for a type A user. It turns out that timely exercise of the promotion option makes more type A users serviced and, therefore, increases the profit. This reflects that Model 3 is more comprehensive than Models 1 and 2 in facility valuation.

\section{CONCLUSION}

In this paper, the layout flexibility in facility management has been explored and its valuation has been modeled and implemented by a quantitative approach based on the MC simulation and integer programming. Through the numerical tests, we demonstrate that effectively and timely exercising the managerial options can significantly increase profit and service quality. Flexibility has value, which can sometimes be optimized. Therefore, flexibility should not be left out when one evaluates the performance of a facility or an infrastructure system. In other words, the definition of performance should incorporate the value of flexibility. 
From the real options perspective, the problem setup in this paper may be viewed as a facility with two switchable and convertible functions. While real options for switching functions have been well studied, functions that are both switchable and convertible have not been identified. It is hoped that this paper can shed some light on design and development of facilities with multiple, switchable, and convertible functions. The proposed approach may also be extended to facility expansion, maintenance and rehabilitation to increase service life and maintain service quality. 


\section{REFERENCES}

Bazaraa M S and Goode J J (1975). "A cutting-plane algorithm for the quadratic setcovering problem." Operations Research 23: 150-158.

Francis R L, McGinnis, L F and White J A (1992). Facility Layout and Location: An Analytical Approach, 2nd edition. Prentice Hall: Englewood Cliffs, NJ.

Heragu S S (1997). Facility Design. PWS Publishing Company: Boston, MA.

Heragu S S and Kochhar J S (1994). "Material Handling Issues in Adaptive Manufacturing Systems." The Materials Handling Engineering Division 75th Anniversary Commemorative Volume, ASME, New York, NY.

Hull J C (1999). Options, Futures, and Other Derivative, 4th edition. Prentice Hall: Englewood Cliffs, NJ.

Kochhar J S and Heragu S S (1999). "Facility layout design in a changing environment." International Journal of Production Research, 11: 2429-2446.

Koopmans T C and Beckmann M J (1957). "Assignment problems and the location of economic activities." Econometrics 25: 53-76.

Lacksonen T A (1994). "Static and dynamic layout problems with varying areas." Journal of the Operational Research Society 45: 59-69.

Love R F and Wong J Y (1976). "On solving a one-dimensional space allocations problem with integer programming." Canadian Journal of Operational Research and Information Processing 14: 139-143.

Luenberger D G (1998). Investment science. Oxford: New York, NY.

Montreuil B (1990). "A modelling framework for integrating layout design and flow network design," In Preprints of Proceedings of the 1990 Material Handling Research Colloquium," 43-58. Material Handling Institute, Hebron, KY.

Trigeorgis L (1996). Real Options: Managerial Flexibility and Strategy in Resource Allocation. The MIT Press: Cambridge, MA. 


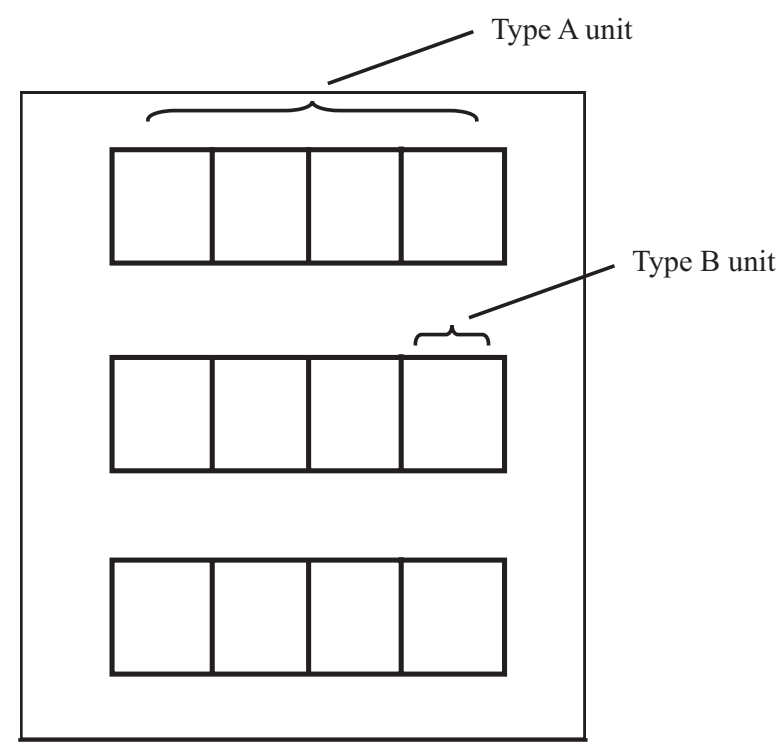

Figure 1: Floor layout of the office building considered

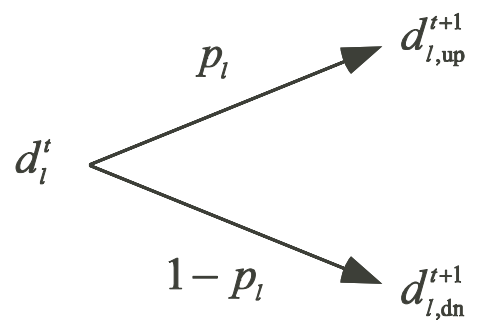

Figure 2: Binomial branching

Table 1: Complexity of the models

\begin{tabular}{ccc}
\hline Model & Number of Variables & Number of Constraints \\
\hline$\left(P_{1}^{t}\right)$ & $2 m_{A} m_{B}+m_{A}$ & $m_{A} m_{B}+2 m_{A}+3$ \\
$\left(P_{2}^{t}\right)$ & $6 m_{A} m_{B}+2 m_{A}$ & $2 m_{A} m_{B}+m_{A}+8$ \\
Two-stage $\left(P_{3}^{t}\right)$ & $\left(6 m_{A} m_{B}+2 m_{A}\right)(S+1)$ & $\left(2 m_{A} m_{B}+m_{A}+8\right)(S+1)$ \\
Three-stage $\left(P_{3}^{t}\right)$ & $\left(6 m_{A} m_{B}+2 m_{A}\right)\left(S^{2}+S+1\right)$ & $\left(2 m_{A} m_{B}+m_{A}+8\right)\left(S^{2}+S+1\right)$ \\
\hline
\end{tabular}


Table 2: Values of the parameters in facility layout numerical tests

\begin{tabular}{cc}
\hline Parameter & Value \\
\hline$R_{A}^{t}$ & $\$ 60,000$ per unit per year \\
$R_{B}^{t}$ & $\$ 10,000$ per unit per year \\
$R_{C}^{t}$ & $\$ 8,000$ per type B unit per year \\
$m_{A}$ & 3 \\
$m_{B}$ & 4 \\
$\mu_{A}$ & 0.05 \\
$\sigma_{A}$ & 0.4 \\
$\mu_{B}$ & 0.05 \\
$\sigma_{B}$ & 0.2 \\
$\mu_{C}$ & 0.05 \\
$\sigma_{C}$ & 0.2 \\
$c_{B}$ & $\$ 1,000$ per unit \\
$r$ & $8 \%$ \\
$T$ & 25 years \\
\hline
\end{tabular}


Table 3: Complexity for $\left(P_{3}^{t}\right)\left(m_{A}=3, m_{B}=4, S=32\right)$

\begin{tabular}{cccc}
\hline Model & Avg. no. of variables & Avg. no. of constraints & Total CPU time (sec) \\
\hline$\left(P_{1}^{t}\right)$ & 17 & 15 & 25 \\
$\left(P_{2}^{t}\right)$ & 42 & 17 & 37 \\
Two-stage $\left(P_{3}^{t}\right)$ & 1386 & 561 & 3674 \\
Three-stage $\left(P_{3}^{t}\right)$ & 31710 & 17969 & $\mathrm{~N} / \mathrm{A}$ \\
\hline
\end{tabular}

Table 4: Total system profit in six different cases with the promotion $\left(\$ 10^{3}\right)$

\begin{tabular}{ccccccc}
\hline$m_{A}$ & $D_{A}^{0}$ & $D_{B}^{0}$ & $D_{C}^{0}$ & Model 1 & Model 2 & Model 3 \\
\hline 3 & 2 & 6 & 3 & 1501 & 1509 & 1602 \\
6 & 4 & 12 & 6 & 3009 & 3084 & 3226 \\
9 & 6 & 18 & 9 & 4523 & 4662 & 4848 \\
12 & 8 & 24 & 12 & 6007 & 6215 & 6460 \\
15 & 10 & 30 & 15 & 7501 & 7789 & 8073 \\
18 & 12 & 36 & 18 & 9002 & 9327 & 9684 \\
\hline
\end{tabular}

Table 5: Total system profit in six different cases without the promotion $\left(\$ 10^{3}\right)$

\begin{tabular}{cccccc}
\hline$m_{A}$ & $D_{A}^{0}$ & $D_{B}^{0}$ & Model 1 & Model 2 & Model 3 \\
\hline 3 & 2 & 6 & 1470 & 1487 & 1502 \\
6 & 4 & 12 & 2956 & 3041 & 3069 \\
9 & 6 & 18 & 4462 & 4601 & 4640 \\
12 & 8 & 24 & 5927 & 6174 & 6232 \\
15 & 10 & 30 & 7426 & 7679 & 7750 \\
18 & 12 & 36 & 8896 & 9216 & 9298 \\
\hline
\end{tabular}


Table 6: The service rate for type A demand with the promotion

\begin{tabular}{ccccccc}
\hline$m_{A}$ & $D_{A}^{0}$ & $D_{B}^{0}$ & $D_{C}^{0}$ & Model 1 & Model 2 & Model 3 \\
\hline 3 & 2 & 6 & 3 & 0.317 & 0.326 & 0.396 \\
6 & 4 & 12 & 6 & 0.318 & 0.349 & 0.401 \\
9 & 6 & 18 & 9 & 0.318 & 0.359 & 0.401 \\
12 & 8 & 24 & 12 & 0.315 & 0.360 & 0.400 \\
15 & 10 & 30 & 15 & 0.314 & 0.362 & 0.399 \\
18 & 12 & 36 & 18 & 0.312 & 0.362 & 0.399 \\
\hline
\end{tabular}

Table 7: The service rate for type B demand with the promotion

\begin{tabular}{ccccccc}
\hline$m_{A}$ & $D_{A}^{0}$ & $D_{B}^{0}$ & $D_{C}^{0}$ & Model 1 & Model 2 & Model 3 \\
\hline 3 & 2 & 6 & 3 & 0.239 & 0.209 & 0.137 \\
6 & 4 & 12 & 6 & 0.240 & 0.208 & 0.145 \\
9 & 6 & 18 & 9 & 0.238 & 0.198 & 0.146 \\
12 & 8 & 24 & 12 & 0.245 & 0.199 & 0.147 \\
15 & 10 & 30 & 15 & 0.243 & 0.196 & 0.150 \\
18 & 12 & 36 & 18 & 0.245 & 0.197 & 0.152 \\
\hline
\end{tabular}

Table 8: The service rate for type $\mathrm{C}$ demand with the promotion

\begin{tabular}{ccccccc}
\hline$m_{A}$ & $D_{A}^{0}$ & $D_{B}^{0}$ & $D_{C}^{0}$ & Model 1 & Model 2 & Model 3 \\
\hline 3 & 2 & 6 & 3 & 0.0375 & 0.0351 & 0.0870 \\
6 & 4 & 12 & 6 & 0.0364 & 0.0349 & 0.0649 \\
9 & 6 & 18 & 9 & 0.0345 & 0.0340 & 0.0585 \\
12 & 8 & 24 & 12 & 0.0354 & 0.0335 & 0.0546 \\
15 & 10 & 30 & 15 & 0.0313 & 0.0284 & 0.0529 \\
18 & 12 & 36 & 18 & 0.0325 & 0.0309 & 0.0529 \\
\hline
\end{tabular}


Table 9: The occupancy rate for type A unit with the promotion

\begin{tabular}{ccccccc}
\hline$m_{A}$ & $D_{A}^{0}$ & $D_{B}^{0}$ & $D_{C}^{0}$ & Model 1 & Model 2 & Model 3 \\
\hline 3 & 2 & 6 & 3 & 0.550 & 0.568 & 0.697 \\
6 & 4 & 12 & 6 & 0.556 & 0.615 & 0.707 \\
9 & 6 & 18 & 9 & 0.557 & 0.631 & 0.709 \\
12 & 8 & 24 & 12 & 0.551 & 0.631 & 0.707 \\
15 & 10 & 30 & 15 & 0.549 & 0.636 & 0.706 \\
18 & 12 & 36 & 18 & 0.546 & 0.635 & 0.704 \\
\hline
\end{tabular}

Table 10: The occupancy rate of type B unit with the promotion

\begin{tabular}{ccccccc}
\hline$m_{A}$ & $D_{A}^{0}$ & $D_{B}^{0}$ & $D_{C}^{0}$ & Model 1 & Model 2 & Model 3 \\
\hline 3 & 2 & 6 & 3 & 0.446 & 0.428 & 0.299 \\
6 & 4 & 12 & 6 & 0.443 & 0.381 & 0.292 \\
9 & 6 & 18 & 9 & 0.442 & 0.367 & 0.290 \\
12 & 8 & 24 & 12 & 0.448 & 0.368 & 0.292 \\
15 & 10 & 30 & 15 & 0.450 & 0.363 & 0.293 \\
18 & 12 & 36 & 18 & 0.453 & 0.361 & 0.295 \\
\hline
\end{tabular}


Table 11: The service rate for type A demand without the promotion

\begin{tabular}{cccccc}
$m_{A}$ & $D_{A}^{0}$ & $D_{B}^{0}$ & Model 1 & Model 2 & Model 3 \\
\hline 3 & 2 & 6 & 0.307 & 0.326 & 0.341 \\
6 & 4 & 12 & 0.313 & 0.350 & 0.354 \\
9 & 6 & 18 & 0.318 & 0.357 & 0.373 \\
12 & 8 & 24 & 0.315 & 0.362 & 0.376 \\
15 & 10 & 30 & 0.318 & 0.360 & 0.375 \\
18 & 12 & 36 & 0.317 & 0.361 & 0.376 \\
\hline
\end{tabular}

Table 12: The service rate for type B demand without the promotion

\begin{tabular}{cccccc}
\hline$m_{A}$ & $D_{A}^{0}$ & $D_{B}^{0}$ & Model 1 & Model 2 & Model 3 \\
\hline 3 & 2 & 6 & 0.250 & 0.230 & 0.217 \\
6 & 4 & 12 & 0.245 & 0.205 & 0.190 \\
9 & 6 & 18 & 0.241 & 0.200 & 0.187 \\
12 & 8 & 24 & 0.244 & 0.195 & 0.181 \\
15 & 10 & 30 & 0.240 & 0.196 & 0.182 \\
18 & 12 & 36 & 0.241 & 0.196 & 0.182 \\
\hline
\end{tabular}


Table 13: The occupancy rate for type A unit without the promotion

\begin{tabular}{cccccc}
$m_{A}$ & $D_{A}^{0}$ & $D_{B}^{0}$ & Model 1 & Model 2 & Model 3 \\
\hline 3 & 2 & 6 & 0.535 & 0.568 & 0.644 \\
6 & 4 & 12 & 0.547 & 0.614 & 0.653 \\
9 & 6 & 18 & 0.557 & 0.628 & 0.652 \\
12 & 8 & 24 & 0.551 & 0.637 & 0.653 \\
15 & 10 & 30 & 0.557 & 0.634 & 0.652 \\
18 & 12 & 36 & 0.554 & 0.634 & 0.651 \\
\hline
\end{tabular}

Table 14: The occupancy rate of type B unit without the promotion

\begin{tabular}{cccccc}
\hline$m_{A}$ & $D_{A}^{0}$ & $D_{B}^{0}$ & Model 1 & Model 2 & Model 3 \\
\hline 3 & 2 & 6 & 0.440 & 0.408 & 0.327 \\
6 & 4 & 12 & 0.432 & 0.367 & 0.329 \\
9 & 6 & 18 & 0.423 & 0.354 & 0.330 \\
12 & 8 & 24 & 0.430 & 0.346 & 0.331 \\
15 & 10 & 30 & 0.425 & 0.347 & 0.330 \\
18 & 12 & 36 & 0.426 & 0.348 & 0.331 \\
\hline
\end{tabular}

